\title{
Another Look at Physicians' Treatment of Men and Women with Common Complaints ${ }^{1}$
}

\author{
Lois M. Verbrugge ${ }^{2,3}$ and Richard P. Steiner ${ }^{4}$ \\ The University of Michigan
}

A recent study of San Diego patients found that men received more extensive and appropriate diagnostic workups than women did for five common complaints (Armitage, K. J., Schneiderman, L. J., \& Bass, R. A. Journal of the American Medical Association, 1979, 241, 2186-2187). This article is a broader analysis of medical care given to men and women for those complaints (fatigue, headache, vertigo/dizziness, chest pain, back pain), and it uses a national survey of ambulatory-care visits. We find that medical care is usually similar for men and women. When significant sex differences do appear, they often show that women receive more medical care during a visit. To some extent, differential care stems from different medical needs that men and women with a complaint have; when some medically relevant factors are controlled, half of the significant sex differences disappear. But half persist, and this suggests that psychosocial factors also underlie differential care for men and women. These may originate with patients (for example, their requests for particular services) or with physicians (for example stereotypes of men and women patients). In contrast to the San Diego study, the national data show that (1) women sometimes receive more diagnostic workups for the five complaints, and (2) when medical factors are controlled, sex differences in the extent and content of workups disappear. The only exception is that men with vertigo/dizziness receive more appropriate workups.

\footnotetext{
'This research was funded by a grant from Hoffman-LaRoche Inc. The authors have full responsibility for the empirical analysis and interpretations.

${ }^{2}$ Institute of Gerontology and Survey Research Center, The University of Michigan, Ann Arbor, Michigan 48109.

${ }^{3}$ To whom correspondence should be addressed at Institute of Gerontology, 300 North Ingalls, The University of Michigan, Ann Arbor, Michigan 48109.

${ }^{4}$ Department of Mathematical Sciences, University of Akron, Akron, Ohio 44325.
} 
The topic of sex differences in medical care has become a strong concern among people who monitor health-care practices, including physicians, legislators, medical sociologists, and consumer advocates. The hypotheses offered to explain sex differences tend to resolve into two basic views: one, that physicians treat men and women differently because of medically relevant factors; and alternatively, that psychosocial factors such as physician stereotypes about patients and patients' help-seeking behavior account for the differential care.

A recent, highly publicized study (Armitage et al,, 1979) reviewed the medical records of 52 married couples who had been served by a San Diego group practice for 5 years or more. The men patients received more extensive and more appropriate workups than the women did for five common complaints. The investigators measured two aspects of diagnostic services: their extent (depth of medical history or examination plus number of diagnostic tests done) and their content (proportion of procedures generally considered appropriate for the complaint which were actually done). For all five complaints, men had higher average scores than women did for both extent and content, with just one exception. However, few of the sex differences were statistically significant ( $p<.05$ for only 2 of the 10 differences). This is partly because the number of people with each complaint was small.

In this article, we examine a large national data set of ambulatory-care visits to see how diagnostic services, therapeutic services, and dispositions for follow-up care differ by sex for the same five complaints. We assess if sex differences in care are due to medically relevant factors or not. In an effort to replicate the findings of Armitage et al. (1979), we create variables about the extent and content of diagnostic services, and we compare the national results with the San Diego results.

\section{METHODS}

\section{Data Source}

The National Ambulatory Medical Care Survey (NAMCS) is a continuous survey of patient visits to office-based physicians in the United States. It has been conducted since 1973 by the National Center for Health Statistics. Each year, a national probability sample of physicians provides special records for patient visits during randomly assigned 7-day reporting periods. We studied NAMCS data for the calendar year 1975, when 2472 physicians participated in the survey. The 1975 Patient Record is presented in Fig. 1.

We used a subsample of the 1975 NAMCS data: visits for five complaints by adults $(18+$ years old) who were old patients presenting new 


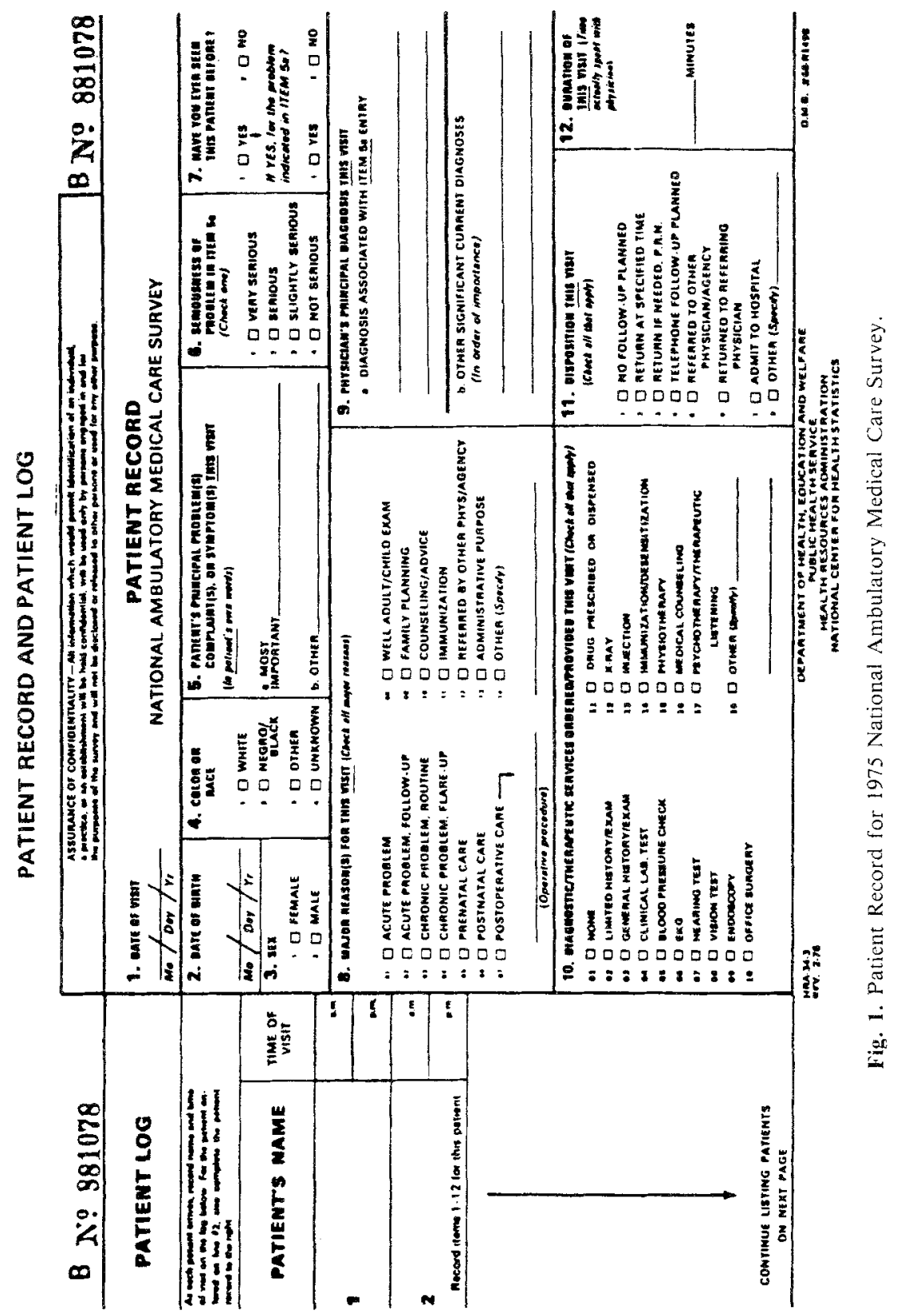


problems. This means that the patients were previously seen by the physician but not for these specific problems. The five complaints are fatigue, headache, vertigo/dizziness, chest pain, and back pain. Up to three complaints are listed in the Patient Record, with the principal one written first. We scanned principal complaints and selected records with the five problems just mentioned. Our subsample included 480 visits by men and 733 visits by women.

All results in this article are based on weighted data. The weighting adjusts for the complex sampling design of NAMCS, so that results are representative of all ambulatory-care visits in the United States during a given year. The proper weights were included on the computer tape obtained from the National Center for Health Statistics. The NAMCS sample design is described in detail elsewhere (Koch \& McLemore, 1978). The physicians' specialties and geographic distribution are also shown in that report.

For simplicity, many statements in this article refer to men and women, but the true units of analysis are men's visits and women's visits.

\section{Variables}

Dependent Variables. Altogether, there were more than 30 dependent variables in the analysis. First, we used the 18 specific services and 8 specific dispositions listed in the Patient Record. They are dichotomous variables $(0=$ not checked, 1 = checked). Second, we constructed several variables from the Patient Record: the total number of services ordered or provided, the total number of dispositions for follow-up care; the depth of the physical examination, and the duration of the visit with the physician. They are identified by these abbreviations: NUMSERV, NUMDISP, EXAM, and DURATION. (EXAM is scored as follows: general exam $=2$, limited exam $=1$, no exam $=0$.) Third, indexes about the extent and content of diagnostic services were created to be parallel to these in the San Diego study. The San Diego researchers provided us information about their coding rules, and we followed them as closely as possible for the NAMCS data. We have extent variables for all five complaints: EXTENT is a count of the diagnostic services checked on the Patient Record, each service scoring 1 except limited exam $=2$ and general exam $=3$. We have content variables for three of the complaints: for headache, CONTENT equals 2 if a blood-pressure check and an eye exam were both done, 1 if one procedure only was done, and 0 if neither was done. For vertigo/dizziness, CONTENT equals 2 if a bloodpressure check and a hearing exam were both done, 1 if one procedure only was done, and 0 if neither was done. For chest pain, CONTENT equals 1 if a blood-pressure check was done and 0 otherwise. The data set did not 
permit content variables for fatigue or back pain which would be similar to the San Diego ones. Overall, the extent variables in the two studies are very similar. The content variables are less so; NAMCS variables are more limited, being based on sparser information than full medical records contain.

In our analysis, all of the dependent variables were treated as interval scaled.

Independent Variables. These were items from the Patient Record that we took to be largely objective criteria in the physicians' evaluation and decisions about treatment: the patient's age, the seriousness of the complaint, whether the patient was sick (ill or injured) or not, the reasons for the visit in the physician's judgment (acute problem, chronic problem, postoperative, other), and the final diagnosis for the principal complaint. Diagnoses were coded according to the International Classification of Diseases (ICDA-8) (National Center for Health Statistics, 1967). We considered the patient ill or injured if any diagnosis in the Patient Record had an ICDA-8 code of 001-999; otherwise, the physician did not find any illness or injury, and the record had codes of Y00-Y13 for all diagnoses.

We refer to the independent variables as "medically relevant factors."

All but one of the independent variables were nominal scaled. In multiple regressions, they were included by using "dummy" variables. (Each category is treated as a separate variable, and a coefficient is estimated for it.) The exception was age, which was coded in single years and was treated as an interval scale variable.

\section{Comparability of the National and San Diego Samples}

The San Diego patients were all long-term clients of the practice, and only their first presentations of the five complaints were analyzed. Our NAMCS subsample was virtually the same, being limited to "old" patients with new problems.

The San Diego sample included only white, middle-class couples. For NAMCS, the Patient Record had no information about socioeconomic status so no control for social class was possible. The record did indicate race but we wanted to preserve the full national representativeness of the data and chose not to restrict the analysis to whites. Actually, over $80 \%$ of the NAMCS visits for the five complaints were made by whites, so the racial composition of our subsample was close to the San Diego sample's.

Table I compares basic characteristics of the two samples. In the national sample, women made the majority of visits for the five complaints $(51-77 \%)$, and they were usually older than the men patients. In the smaller San Diego sample, men made the majority of visits for three complaints, 


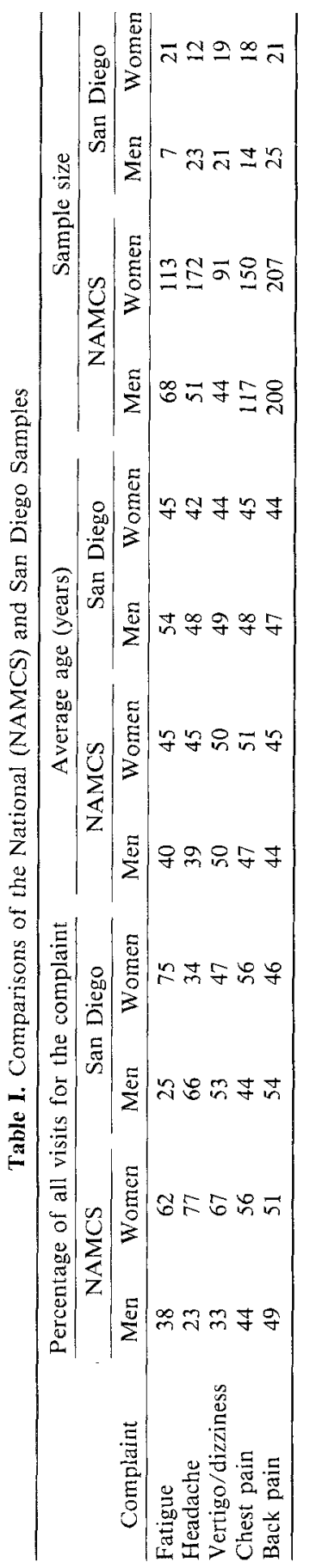


and they were older than the women for all five. This is partly because the sample was based on married couples, and husbands tend to be older than their wives. Thus, the San Diego sample is quite different from the national one. The age differences influence the results: older people tend to receive more care during an office visit. This boosts men's care in the San Diego study and women's in the national one. To remove this effect, we controlled for age throughout the NAMCS analysis. (It is not controlled in the San Diego analysis, so the boosting effect of age on men's care remains.)

\section{Statistical Analysis}

Descriptive analysis was done to show how men and women differ on the independent and dependent variables. Then regression analyses were conducted using three models: Model A is $\hat{Y}=f[\mathrm{Sex}]$. It shows the zero-order (initial) effect of sex on medical care. Model B is $\hat{Y}=f[$ Age, Seriousness, If Ill or Injured, Reasons for Visit, Sex]. It shows the effect of sex after controlling for medically relevant factors. Model $\mathrm{C}$ is a modification of Model B. To give a more specific control for the physician's medical evaluation, it replaces "If Ill or Injured" with the final diagnosis. Model C is therefore $\hat{Y}=f$ [Age, Seriousness, Final Diagnosis, Reasons for Visit, Sex]. Models $B$ and $C$ answer the key question of this article: Are initial sex differences in physicians' care due to medical differences of men and women or not? In other words, do significant sex differences in care disappear (become nonsignificant) once we control for medically relevant factors? [A note of caution to readers: In another article on the NAMCS data (Verbrugge \& Steiner, 1981), the models labeled $B$ and $C$ here are labeled $C$ and $C 1$.

\section{RESULTS}

We first describe how men and women patients differ in their presenting characteristics (age, seriousness, etc.) and whether physicians' care is related to those characteristics. Then we analyze how often physicians give differential care to men and women patients and how well this can be explained by medically relevant factors.

\section{Presenting Characteristics That Influence Medical Care}

Men and women with the five complaints differ as follows: men have more serious $(p<.01)$ and acute $(p<.01)$ headaches, but women with headaches are older $(p<.05)$ (see Table II). Similarly, men have more seri- 


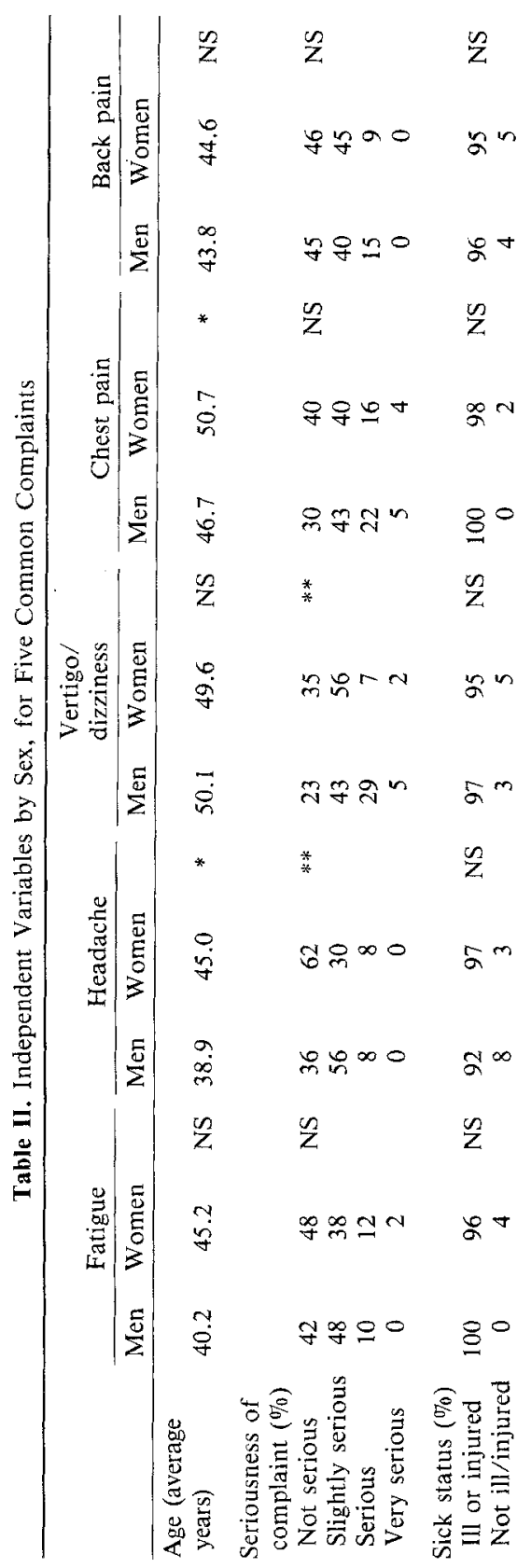




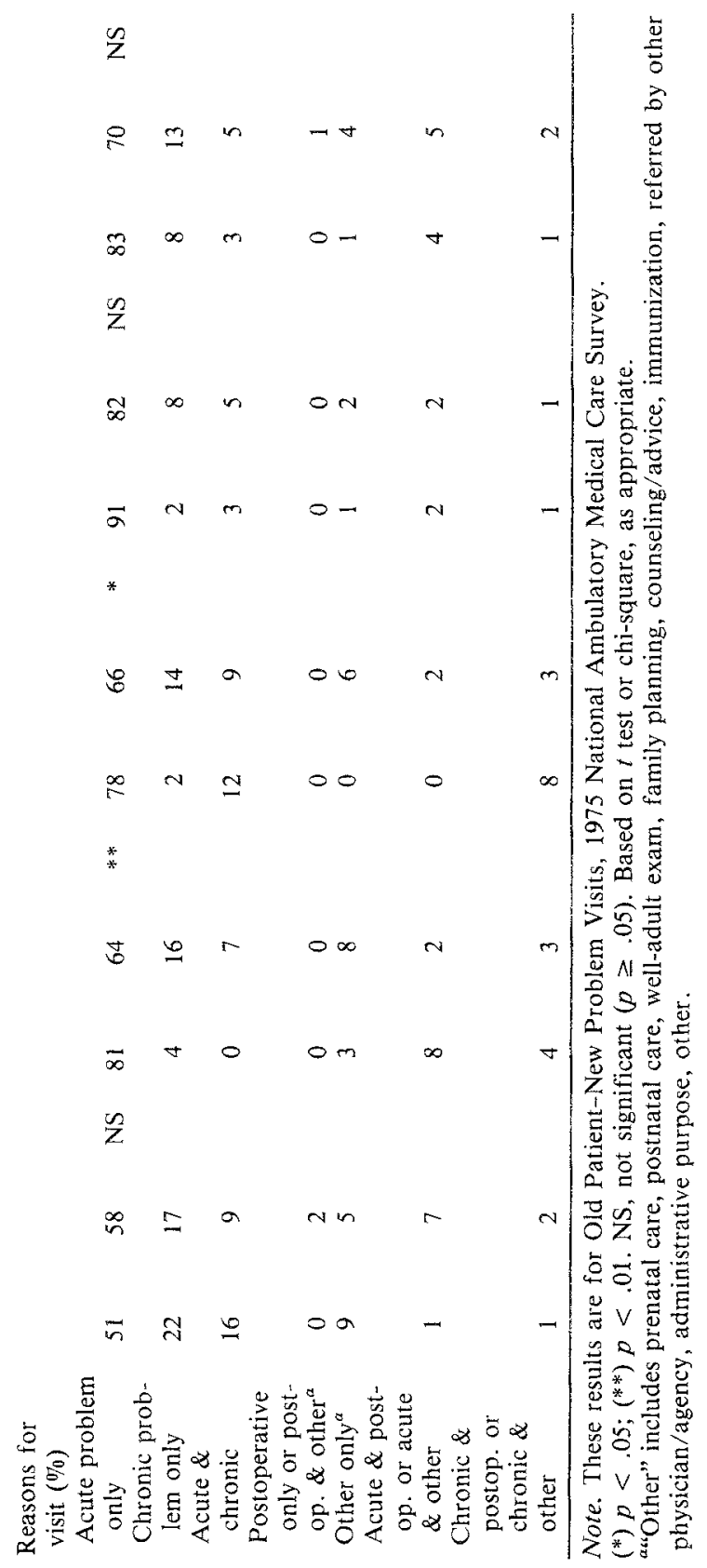


ous $(p<.01)$ vertigo/dizziness, and it is more often linked to acute or postoperative problems $(p<.05)$ for them. Women with chest pain are older $(p<.05)$ than men with this complaint. Other sex differences are visible in Table II (e.g., women with fatigue are older, and men have more serious chest and back pain), but they are not statistically significant.

Several characteristics of the patients prompt more medical care from their physicians: older age, more serious symptoms, a diagnosis of illness or injury, and acute problems tend to prompt more services (data not shown). Follow-up care is more common for older patients, more serious symptoms, ill or injured patients, and chronic problems. We have found these same relationships for the entire NAMCS sample of adult visits too (Verbrugge \& Steiner, 1981).

\section{Sex Differences in Physician Care}

Table III shows the services and dispositions that occur significantly more for men or women. The top part of Table IV states these differences in regression form (Model A).

The tables show that men and women who complain of fatigue, headache, vertigo/dizziness, chest pain, and back pain usually receive similar medical care. Overall, $77 \%$ of the comparisons (121 of 157 sex differences) are not statistically significant. Of the 36 significant differences, about half (17) indicate that women get more care, and half (19) that men do. Significantly more services are provided to women who complain of headache or chest pain and to men who complain of vertigo/dizziness. For fatigue and back pain, men sometimes receive significantly more services; sometimes women do. With regard to dispositions, women are often asked to return when they complain of fatigue or headache. Men with chest pain or back pain are more often admitted to the hospital.

\section{Sex Differences in Care After Controlling for Some Medically Relevant Factors}

When the medically relevant factors described earlier are taken into account (Model B), one-third of the initial sex differences lose their significance (11 of 36 , or $31 \%$ ) (see second part of Table IV). Most (nine) of these pertain to headache, vertigo/dizziness, and chest pain. These are the complaints for which men and women patients differ most in their presenting characteristics.

Nevertheless, the majority (25) of initial differences persist, and their strength is undiminished by the controls. To try to account for these differences, we included a specific control for the final diagnosis (Model C). We 


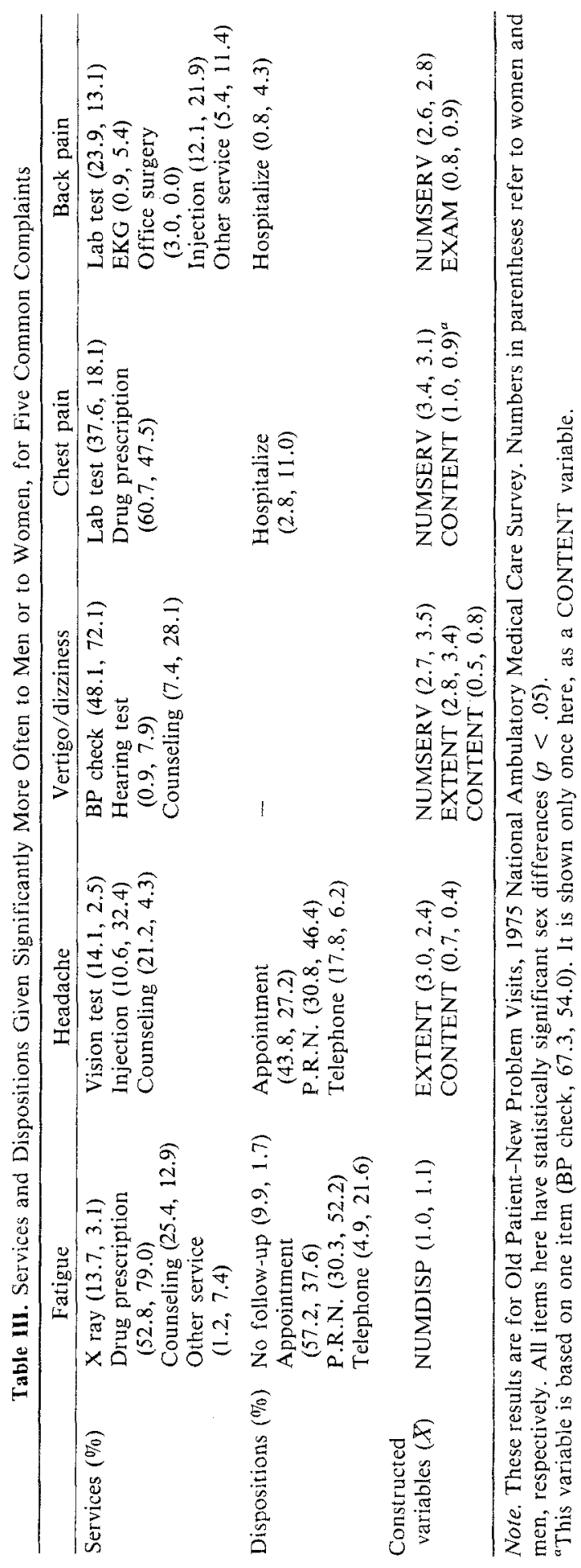




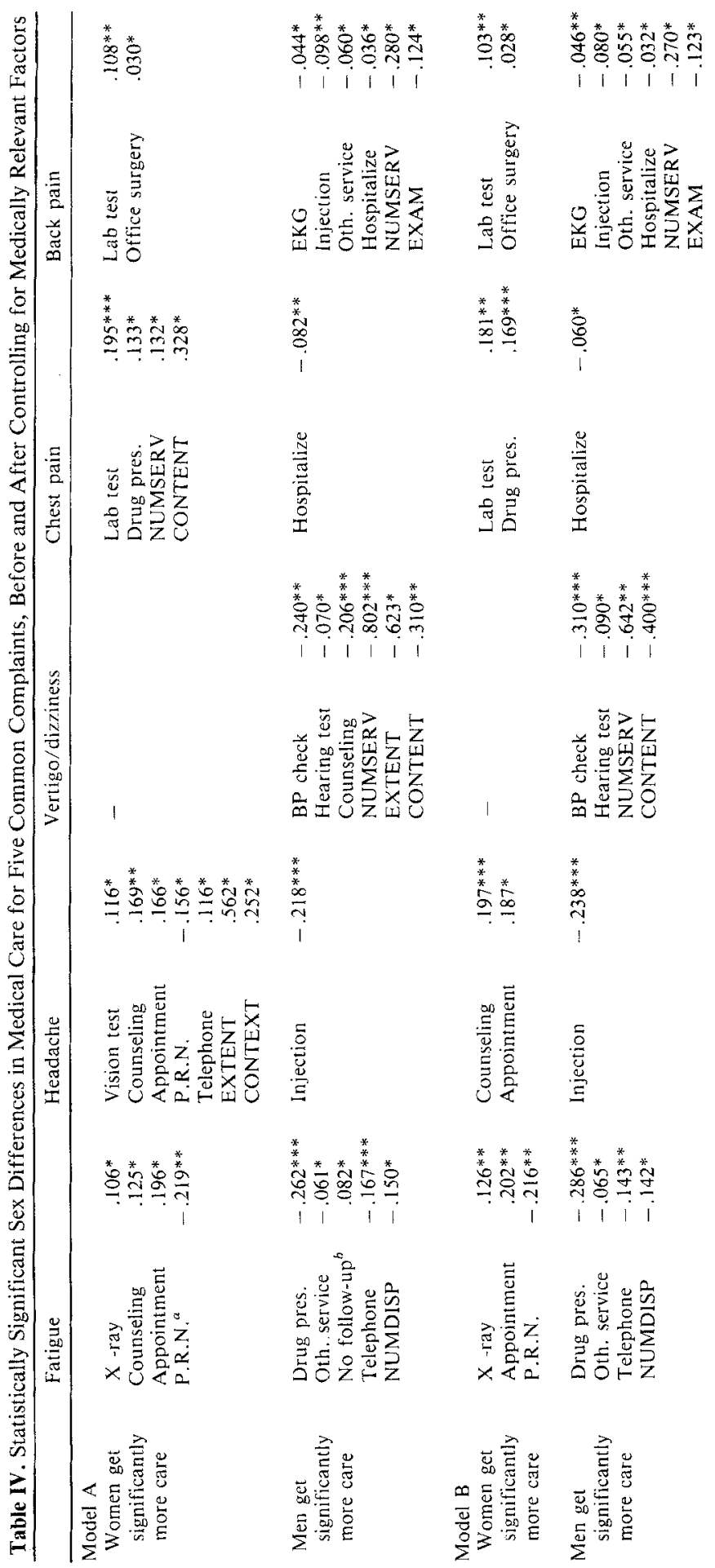




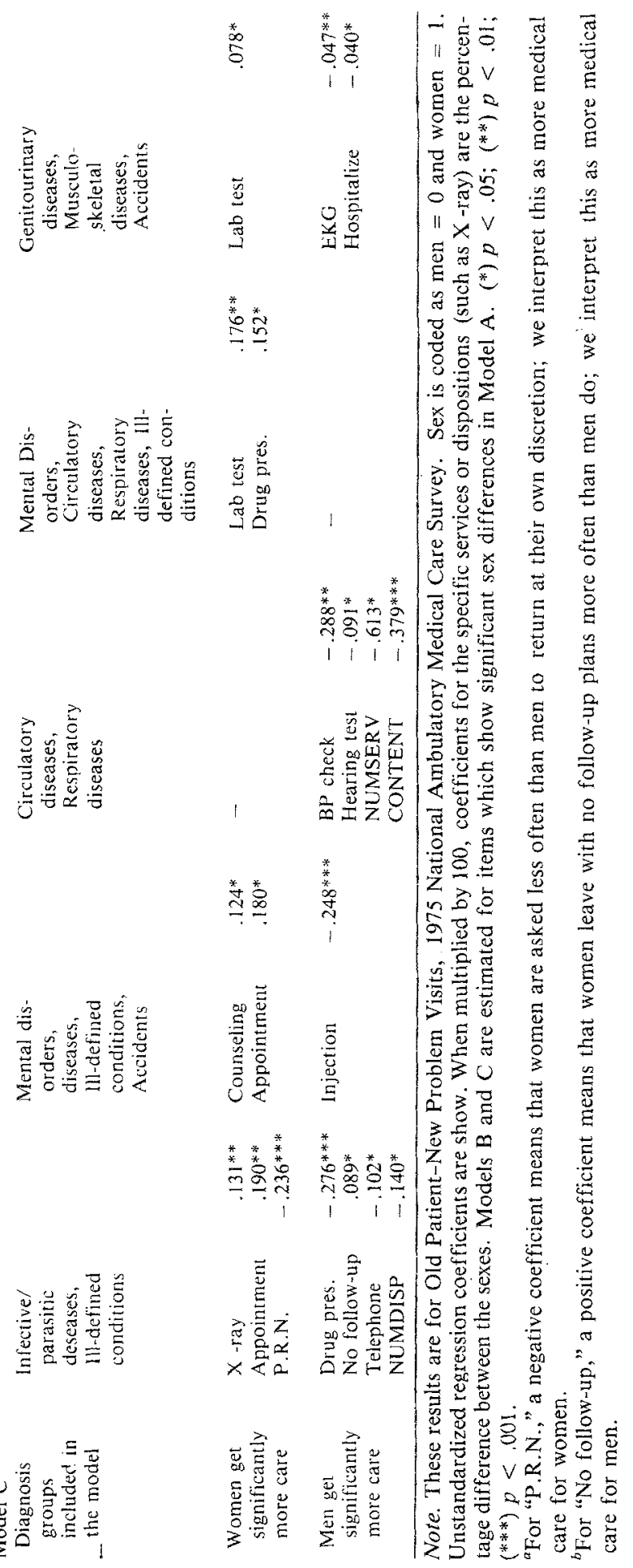


Table V. Sex Differences in Final Diagnoses, for Five Common Complaints

\begin{tabular}{|c|c|c|}
\hline \multirow[b]{2}{*}{ Complaint } & \multicolumn{2}{|c|}{ Diagnoses received more often for } \\
\hline & Men's visits & Women's visits \\
\hline Fatigue & $\begin{array}{l}\text { Infective/parasitic diseases, } \\
\text { esp. mononucleosis }(2.9 \%, 8.2 \%) \\
\text { Ill-defined conditions, esp. nervous- } \\
\text { ness and debility }(16.0,32.8)\end{array}$ & - \\
\hline Headache & $\begin{array}{l}\text { Respiratory diseases, esp. } \\
\text { influenza }(23.6,42.4) \\
\text { Accidents }(3.7,14.3)\end{array}$ & $\begin{array}{l}\text { Mental disorders }(23.8,3.8) \\
\text { Ill-defined conditions, esp. } \\
\text { headache }(19.5,9.5)\end{array}$ \\
\hline $\begin{array}{l}\text { Vertigo/ } \\
\text { dizziness }\end{array}$ & $\begin{array}{l}\text { Circulatory diseases } \\
(18.8,34.0)\end{array}$ & $\begin{array}{l}\text { Respiratory diseases, esp. } \\
\text { chronic sinusitis }(13.6,7.1)\end{array}$ \\
\hline Chest pain & $\begin{array}{l}\text { Circulatory diseases, esp. } \\
\text { myocardial infarction and } \\
\text { angina }(14.9,26.9) \\
\text { Ill-defined conditions } \\
(9.8,17.9)\end{array}$ & $\begin{array}{l}\text { Mental disorders, esp. } \\
\text { neuroses }(6.0,1.0) \\
\text { Respiratory diseases, esp. } \\
\text { bronchitis and pluerisy } \\
(27.9,13.5)\end{array}$ \\
\hline Back pain & Accidents $(33.2,50.5)$ & $\begin{array}{l}\text { Genitourinary diseases. esp. } \\
\text { urinary tract infections }(11.3,1.6) \\
\text { Musculoskeletal diseases, esp. } \\
\text { rheumatism and vertebrogenic } \\
\text { pain syndrome }(35.3,27.3)\end{array}$ \\
\hline
\end{tabular}

Note. These results are for Old Patient-New Problem Visits, 1975 National Ambulatory Medical Care Survey. Numbers in parentheses refer to women and men, respectively. A diagnosis group is listed here if one sex receives substantially more such diagnoses for a given complaint than the other sex (sex difference, $\geq 5 \%$ ).

reasoned that if men and women have ditterent nealth problems underlying a given complaint then different medical care will naturally result. Actually, the NAMCS data show that men and women normally receive similar arrays of diagnoses for a complaint. Table $\mathrm{V}$ shows the diagnostic differences that do occur. Including controls for these diagnostic groups, some improvement in explaining sex differences occurs (see third part of Table IV). Seven more of the remaining sex differences are eliminated (so $p>.05$ ), and another five have their significance level weakened (but still below .05). Most of the eliminated differences pertain to men's greater care for back pain, confirming that their higher prevalence of injuries (Table V) prompts more medical attention for them.

Looking at the significant differences that remain in Model C, 8 indicate more care for women and 11 for men. (The total is 19 because one difference attained significance in Model $\mathrm{C}$ but not Model B.)

In summary, controlling for some medically relevant factors explains $17(47 \%)$ of the initial sex differences in medical care for five common complaints. The controls are especially successful in eliminating differences in care for headache, chest pain, and back pain. This means that differential care for men and women who complain of these problems is often due to sex 


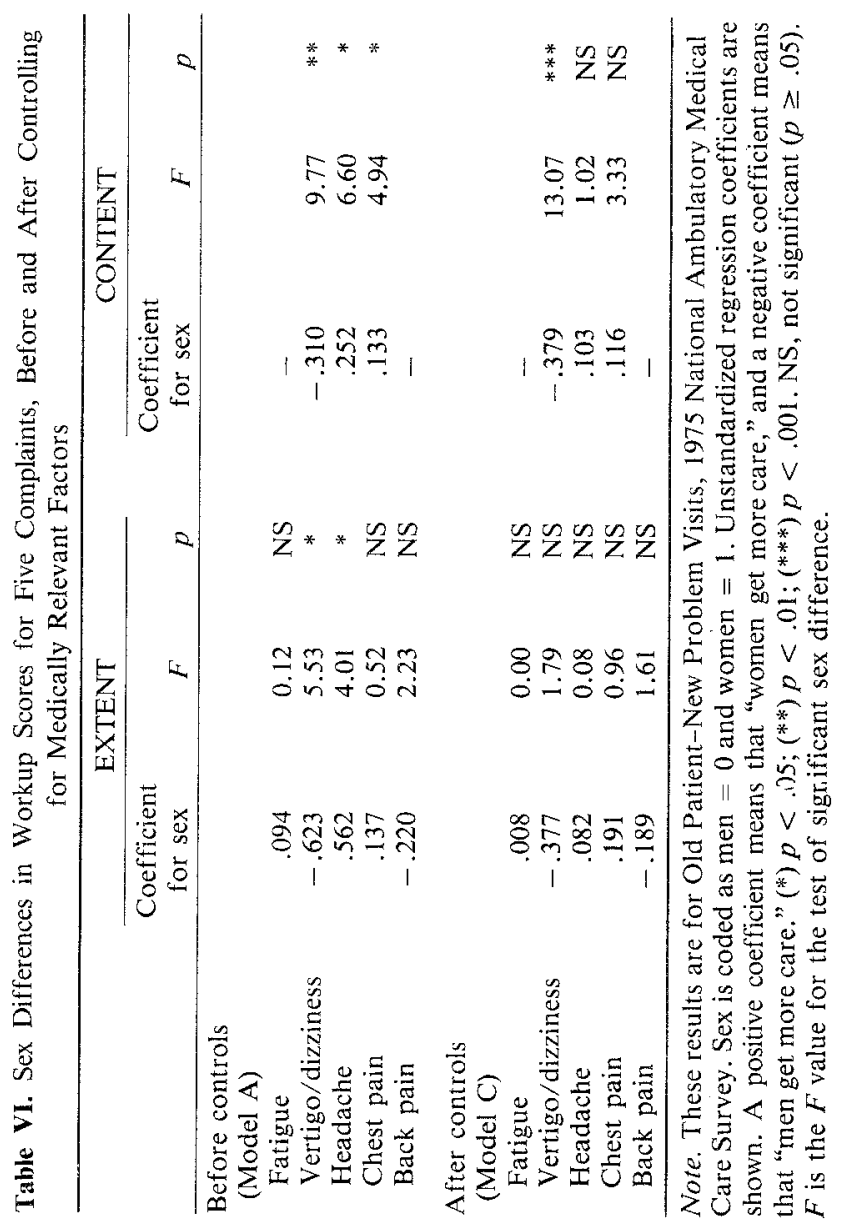


differences in age, seriousness, sick status, diagnosis, and the acute or chronic nature of the problem. Specifically, physicians give more care to women with headaches because the women are older and report more chronic problems than men with headaches. More in-office care to women with chest pain is explained by the same factors. But men with chest pain are hospitalized more often because physicians suspect circulatory diseases. Physicians give more care to men with back pain because their pain is often related to accidents, which require prompt attention.

The controls are less successful in eliminating sex differences in care for fatigue and vertigo/dizziness. Men still get more drug prescriptions and more follow-up plans (except specific appointments) for fatigue, and they get more physician services for vertigo/dizziness.

\section{Comparison with the San Diego Results}

In contrast to the San Diego study, the NAMCS data provide virtually no evidence that men receive more extensive and appropriate workups for the five targeted complaints. Table VI shows NAMCS results for the extent and content variables in a format parallel to that of the San Diego article (Armitage et al., 1979, Table 2).

Before controls (Model A), significant sex differences in diagnostic workups are common (five of eight comparisons); three of these show more care for women. Looking at all eight sex differences (whether significant or not), five show more care for women. The smaller San Diego sample produced few significant differences ( 2 of 10 comparisons); they showed more care for men. Of the 10 total sex differences, virtually all (9) showed more care for men too.

After controls are included for NAMCS (Model C), only one significant sex difference remains, namely, that men receive more appropriate diagnostic care for vertigo/dizziness (see CONTENT in Table VI). This does agree with the San Diego results. But the other four significant differences diminish and become nonsignificant.

In sum, the NAMCS analysis shows that women sometimes get more diagnostic workups for the common complaints (Model A) and that medical factors often explain why one sex receives more care than the other (compare Models A and C).

\section{DISCUSSION}

These results indicate that men and women generally receive similar services and dispositions for their complaints of fatigue, headache, verti- 
go/dizziness, chest pain, and back pain. But there are some significant differences; half of these show more care for women, and half for men. Many of the differences can be explained by medical factors that physicians consider (patient age, seriousness of the complaint, whether the patient is ill/injured or not, final diagnosis, acute versus chronic symptoms). These often differ for men and women patients, and because they naturally influence medical decisions, they help account for differences in treatment given to men and women. Controlling for these medically relevant factors reduces the number of sex differences by about half.

Still, some intriguing differences remain significant in the 1975 NAMCS data. Why do men with fatigue receive more drug prescriptions and women with fatigue more $X$ rays? Why are follow-up plans more numerous and more flexible (telephone, P.R.N.) for fatigued men, whereas fatigued women get more fixed appointments for further care? Why do women with headaches receive more verbal counseling, and men more injections? And why are women with headaches asked to schedule a return visit more often? Why do men with vertigo/dizziness get more total services and more appropriate diagnostic ones? Why do women with chest pain receive more lab tests and drug prescriptions and also more lab tests for their back pain? Why do men with back pain get EKGs more often, and why are they hospitalized more even after taking their higher incidence of injuries into account? These specific differences may or may not appear in other data sets or even other years of NAMCS. The important point is that some sex differences in care do persist and are quite impervious to controls for medical factors.

There are four possible explanations for such differences. The first is a medical reason, and the other three are psychosocial ones. (1) Some important medical factors may still be missing in our analysis (Models $\mathrm{B}$ and $\mathrm{C}$ ). Physicians consider many factors besides age, seriousness, sick status, diagnosis, and reasons for visit. When interpreting a complaint and choosing appropriate treatment, they use their general medical knowledge of disease prevalence, risk factors, and physiology and also their specific knowledge of a patient's medical history. To the extent that men and women have different risks or morbidity associated with the same complaint, differential care should be expected. NAMCS data take a few such factors into account, but certainly not all. (2) Patients often request certain services and follow-up strategies, and physicians may provide these even in the absence of a clear medical rationale. If men and women differ in their requests, this too helps explain differential care. (3) For a given symptom, some patients become very distressed, while other remain calm. Physicians are sensitive to emotional distress and probably offer some services and dispositions to help relieve it. In assessing distress, they take note of gestures, voice tone, elaborateness of symptom descriptions, and mentions of personal troubles. If men and women differ in these signs of emotional distress, this can further account 
for differential care they receive. (4) Physicians may be sex biased, seeing men and women patients in stereotypic ways and basing treatment on those stereotypes rather than responding to each patient's particular problems. For example, if physicians assume that women have more free time than men do, they may schedule return appointments more for women.

The relative importance of these explanations is not yet known. We have controlled for medical factors as far as NAMCS data permit. They prove to be important, but incomplete, reasons for differential care. The data set cannot reveal whether the remaining sex differences are due to still other medical factors or to psychosocial ones.

To date, there has been little research on the psychosocial factors just mentioned. A recent study (Bernstein $\&$ Kane, 1981) shows that physicians respond to patients' expressiveness (whether they discuss a personal problem as well as the physical one) and that patient sex modifies the professional response. Various strategies are being used to study physician sex bias, including experimental vignettes, retrospective analysis of medical records, and tape-recorded conversations between physicians and patients (see Abramowitz \& Dokecki, 1977; Davidson \& Abramowitz, 1980; McCranie et al., 1978; Verbrugge \& Steiner, 1981; Wallen et al., 1979).

Our analysis of medical records for a national sample did not confirm the results of a smaller San Diego study (Armitage et al., 1979). We found that women - not men - often receive more diagnostic workups. Medical factors prove to be an important reason for the differential workups (whether it was women or men who received more care); sex differences diminished when medical characteristics of women and men patients were taken into account. We tend to place more confidence in the NAMCS results because they are based on a large national sample. Some caution is needed, however, because we could not provide perfectly the extent and content variables used in San Diego.

Despite their different results, the two studies have compatible theoretical and research implications. The San Diego researchers suggested three reasons for their results. Two pointed to medically relevant factors: (1) that physicians know more about the medical history of patients who visit frequently and (2) that physicians use their knowledge about men's and women's risks of life threatening diseases and scrutinize symptoms more when risks are high. (3) Another reason pointed to physician sex bias (that physicians take men's symptoms more seriously than women's and offer more thorough care to men). In this analysis, we have suggested additional reasons for sex differences: (4) the medically relevant factors of patient age, seriousness of the complaint, sick status, final diagnosis, and acute or chronic nature of the symptoms; (5) patient requests for specific treatment or follow-up; and (6) patient emotional distress. We have also noted that physician sex bias can sometimes lead to more services and dispositions for women, rather than fewer. Together, 
the two studies suggest an array of plausible reasons for differential medical care given to men and women who present the same complaint.

The topic of sex differences in medical care often sends the popular press rushing to a single explanation, namely, physician sex bias. Although such bias may exist, it is hardly the sole reason for differential care of men and women patients. There are other very likely, and less incendiary, reasons for the differences. Until they are more definitively tested, sex differences in medical care should be interpreted with caution.

\section{REFERENCES}

Abramowitz, C. V., \& Dokecki, P. The politics of clinical judgment: Early empirical returns. Psychological Bulletin, 1977, 84, 460-476.

Armitage, K. J., Schneiderman, L. J., \& Bass, R. A. Response of physicians to medical complaints in men and women. Journal of the American Medical Association, 1979, 241, $2186-2187$.

Bernstein, B., \& Kane, R. Physician attitudes toward female patients. Medical Care, 1981, 19, 600-608.

Davidson, C. V., \& Abramowitz, S. I. Sex bias in clinical judgment: Later empirical returns. Psychology of Women Quarterly, 1980, 4, 377-395.

Koch, H., \& McLemore, T. The National Ambulatory Medical Care Survey: 1975 Summary. Vital and Health Statistics, Series 13, No. 33. Hyattsville, Md.: National Center for Health Statistics, 1978.

McCranie, E. W., Horwitz, A. J., \& Martin, R. M. Alleged sex-role stereotyping in the assessment of women's physical complaints: A study of general practitioners. Social Science and Medicine, 1978, 12, 111-116.

National Center for Health Statistics. Eighth Revision International Classification of Diseases, Adapted for Use in the United States, PHS Publ. No. 1693. Washington D. C.: Government Printing Office, 1967.

Verbrugge, L. M., \& Steiner, R. P. Physician treatment of men and women patients - Sex bias or appropriate care? Medical Care, 1981, 19, 609-632.

Wallen, J., Waitzkin, H., \& Stoeckle, J. D. Physician stereotypes about female health and illness: A study of patient's sex and the informative process during medical interviews. Women and Health, 1979, 4, 135-146. 